\title{
Patient preferences at ten years following initial diagnosis of atrial fibrillation: the Belgrade Atrial Fibrillation Study
}

This article was published in the following Dove Press journal:

Patient Preference and Adherence

23 August 2013

Number of times this article has been viewed

\author{
Tatjana S Potpara ${ }^{1,2}$ \\ Marija M Polovina ${ }^{2}$ \\ Nebojsa M Mujovic ${ }^{1,2}$ \\ Aleksandar M Kocijancic ${ }^{2}$ \\ Gregory YH Lip ${ }^{3}$
}

'Faculty of Medicine, University of Belgrade, ${ }^{2}$ Cardiology Clinic, Clinical Center of Serbia, Belgrade, Serbia; ${ }^{3}$ University of Birmingham Centre for Cardiovascular Sciences, City Hospital, Birmingham, UK
Correspondence: Tatjana S Potpara Cardiology Clinic, Clinical Center of Serbia, Visegradska 26,

II 000 Belgrade, Serbia

Tel +381113616319

Fax +38I II3616318

Email tanjapotpara@gmail.com
Background: Many atrial fibrillation (AF) patients have a poor understanding of the management of this condition. We investigated patient attitudes towards AF and a potential invasive treatment following an average 10-year period of prospective rhythm control in a cohort of newly diagnosed AF patients.

Methods: This was a prospective registry-based study. At the regular annual visit in 2007, patients were asked at random to answer several AF-related questions.

Results: Of 390 patients, 277 (71.0\%) reported symptom reduction over time, but only $45(11.5 \%)$ reported that they had "got used" to AF; 201 patients $(51.5 \%)$ stated they would always prefer sinus rhythm, and $280(71.2 \%)$ would accept an invasive AF treatment. Independent predictors for choosing an invasive procedure were younger age, impaired career/working capacity, and male gender (all $P<0.05$ ).

Conclusion: Our findings suggest that most AF patients prefer sinus rhythm and would readily accept an invasive procedure if it offered the possibility of a cure for their AF.

Keywords: atrial fibrillation, catheter ablation, treatment, symptoms, patient preferences

\section{Introduction}

Atrial fibrillation (AF) is associated with increased morbidity and mortality and impaired quality of life. ${ }^{1-3}$ Randomized trials have failed to demonstrate the superiority of pharmacologic rhythm control over the rate control strategy, and this has been driven largely by the adverse effects of the antiarrhythmic drugs available. ${ }^{4-10}$ Of note, an observational study with a relatively large cohort suggested a beneficial long-term effect of the rhythm control strategy on mortality. ${ }^{11}$ Nonpharmacologic methods for rhythm control are developing rapidly, and catheter ablation is increasingly being used as a first-line treatment, even in patients with chronic AF and advanced structural atrial (and ventricular) alterations. The available data suggest that AF ablation is superior to antiarrhythmic drugs in maintaining sinus rhythm and results in better symptom relief and exercise tolerance. ${ }^{12,13}$ The number of AF patients eligible for ablation is increasing rapidly, but the procedure is still relatively rarely utilized as a first-line treatment option, mostly due to a lack of data from randomized trials. ${ }^{14}$

The increasing availability of catheter ablation for AF could well facilitate reinforcement of AF as a readily modifiable cardiovascular risk factor. ${ }^{13} \mathrm{~A}$ recent analysis of RECORDAF (REgistry on Cardiac rhythm disORDers assessing the control of Atrial Fibrillation), a real-life cohort of AF patients from Europe, America, and Asia, demonstrated that physicians generally prefer a rhythm control strategy, and the most commonly stated reasons for this preference included the restricted nature of patient 
enrollment and the clinical applicability of conclusions drawn from the trials (indeed, the largest of the rhythm versus rate control trials, ie, AFFIRM [Atrial Fibrillation Follow-up Investigation of Rhythm Management], enrolled only 55\% of the patients screened). ${ }^{4,15}$ A recent survey conducted by the AF-AWARE (Atrial Fibrillation AWareness And Risk Education) group found many AF patients to have an insufficient level of understanding of AF, leading to a poor appreciation of AF-related risks and a poor understanding of its management and treatment. ${ }^{16}$

We investigated patient attitudes towards this rhythm disorder and a potential invasive AF treatment following an average 10-year period of prospective active pharmacologic rhythm control treatment in a cohort of middle-aged patients with a new diagnosis of nonvalvular AF.

\section{Patients and methods}

\section{Patient selection and study design}

We conducted an observational study of a subgroup of patients with newly diagnosed AF in the Belgrade Atrial Fibrillation Study registry. This is a prospectively completed registry of patients with nonvalvular AF seen in the Clinical Centre of Serbia between 1992 and 2007. ${ }^{17-19}$ This is the main cardiology center for specialist arrhythmia services, serving the population of Belgrade and the rest of the country. All patients gave their written informed consent to be included in the registry.

Detailed diagnostic evaluation was performed to exclude acute causes of AF and to confirm any underlying comorbid disease. History, physical examination, 12-lead electrocardiogram, blood pressure measurement, blood and urine analysis, chest radiography, and transthoracic echocardiography were performed routinely. Stress testing, coronary angiography, and other procedures were used if needed. A detailed medical history, evaluation of previous medical records and thorough baseline medical examination were used to determine the presence of cardiac or non-cardiac diseases. Patients with acute causes of AF (eg, acute myocardial infarction, hyperthyroidism, fever, severe anemia), ventricular pre-excitation, atrial flutter, valvular heart disease, prosthetic valves, a history of rheumatoid fever, known malignancy, or any advanced serious chronic disease were excluded. Following analysis of detailed medical records for each patient, baseline AF was classified as paroxysmal, persistent, or permanent. ${ }^{14}$

\section{Treatment}

At baseline, rhythm control was attempted in all patients. Further management was at the discretion of the attending cardiologist but the basic approach was to make every reasonable effort to maintain rhythm control. Permanent AF was accepted only if electrocardioversion had failed, AF had persisted for more than one year at any point of follow-up (with left atrial anteroposterior diameter $>55 \mathrm{~mm}$ ), long-term pharmacotherapy for rhythm control had been exhausted or caused complications, or frequent persistent AF episodes were noted despite active treatment.

Cardioversion was attempted using intravenous propafenone, amiodarone, or oral quinidine sulfate, and/or external DC shock. Maintenance of sinus rhythm included intermittent or long-term use of beta blockers or class IC/III drugs in a "stepwise" fashion; in the absence of contraindications, beta blockers or propafenone was tried first, followed by flecainide or sotalol, and finally amiodarone in the event of stubborn recurrent AF. However, patients with impaired left ventricular systolic function received amiodarone (or amiodarone plus a beta blocker) as a first-line option. By the end of follow-up, catheter ablation for AF was not available in our hospital.

In permanent $\mathrm{AF}$, rate control aiming for a target heart rate of $<100$ beats per minute while resting and $\leq 120$ beats per minute during usual activities was attempted either by drugs (digitalis, verapamil, diltiazem, beta blockers) or radiofrequency catheter ablation of the AV node with permanent pacemaker implantation. Aspirin or oral anticoagulants were prescribed for the prevention of thromboembolism according to the guideline recommendations.

\section{Follow-up and questionnaire}

Follow-up lasted for at least 5 years or until death, and patients were reassessed at least annually. At baseline (ie, time of initial diagnosis of AF and inclusion in the registry), each patient received a detailed explanation of the nature, course, and possible complications of AF and AF-related treatment goals, and was encouraged to ask questions and participate actively in the management of AF during followup. At the annual follow-up visit in 2007, patients who had completed at least 5 years of follow-up were randomly invited to answer (and sign) AF-related questions (please see below).

Patients were also asked to state their level of education, employment status, marital status, and whether they had children. Patients were allowed to ask for an additional explanation of the questions, but were not helped regarding the answers. Randomization was based on a systematic sample with selection step 3 plus an additional $10 \%$ of patients to compensate for the possibility of missing data. All patients 
who completed the questionnaire signed at the end of the document.

\section{Statistical analysis}

Following a test of statistical normality, continuous variables are presented as means \pm standard deviations. Categorical variables are reported as counts with percentages. The Student's $t$-test was used for comparison of age, duration of follow-up, and number of AF-related hospitalizations between patients who completed the questionnaire and those who did not. Differences in categorical variables were tested using the chi-square test. Univariate and multivariable stepwise logistic regression analyses were used to assess the relationship between the patients' answers and the clinical characteristics shown in Tables 1 and 2. The association of each of the variables from the tables with the patients' answers was first tested in univariate analyses, and only variables with a significant association $(P<0.05)$ were entered into multivariable models, wherein the dependent variable was "Yes to intervention" or "No to intervention" (Table 4). A $P$-value $<0.05$ was considered to be statistically significant. Statistical analysis was performed using Statistical Package for the Social Sciences version 19 software (SPSS Inc, Chicago, IL, USA).

\section{Results}

Of 3,467 consecutive patients with nonvalvular AF, $1,058(30.5 \%)$ had newly diagnosed AF. We randomly sur- veyed 390 of these 1,058 patients $(36.9 \%)$ at their regular annual follow-up visit during 2007. All randomly selected patients responded to the survey. Clinical characteristics of the patients at the time of survey are shown in Table 1. There were no significant differences between patients in the questionnaire group compared with the rest of the newly diagnosed AF cohort, except for slightly more male patients, a greater prevalence of hypertension, and more frequent use of oral anticoagulants in the questionnaire group $(P<0.05$, Table 1$)$. The mean duration of follow-up was $11.2 \pm 6.9$ years. All patients completed a minimum 5-year follow-up, and 251 patients $(23.7 \%)$ were lost to further follow-up beyond 5 years. Of 390 patients who completed the questionnaire, 361 (92.6\%) reported experiencing AF-related symptoms, and 247 (63.3\%) stated that they had at least three symptoms attributable to AF (Figure 1). The most common complaint was impaired exercise tolerance (270 patients, $69.2 \%$ ), followed by palpitations (244 patients, 62.6\%), occasional very fast heart beat (223 patients, $57.2 \%$ ), fatigue (173 patients, 44.4\%), shortness of breath (133 patients, $34.1 \%$ ), chest pain (119 patients, 30.5\%), dyspnea (99 patients, 25.4\%), dizziness ( 95 patients, $24.5 \%$ ), ankle edema (71 patients, $18.2 \%$ ), syncope ( 25 patients, $6.4 \%$ ), and other symptoms (78 patients, $20.0 \%$ ). Patients' self-reported social characteristics (ie, level of education, employment status, and marital status) are shown in Table 2.

The questions and patients' answers are shown in Table 3. When asked if there was any reduction in

Table I Clinical characteristics of patients at the time of the questionnaire and comparison with patients who filled the questionnaire and those who did not

\begin{tabular}{|c|c|c|c|c|}
\hline Variable n (\%) & $\begin{array}{l}\text { All patients } \\
1058\end{array}$ & $\begin{array}{l}\text { Questionnaire (+) } \\
390(36.9)\end{array}$ & $\begin{array}{l}\text { Questionnaire (-) } \\
668(63.1)\end{array}$ & $P$-value \\
\hline Age at baseline (years) & $52.5 \pm 12.2$ & $51.8 \pm 11.9$ & $52.9 \pm 12.4$ & 0.148 \\
\hline Male gender & $688(65.0)$ & $274(70.3)$ & $4 \mid 4(62.0)$ & 0.007 \\
\hline Hypertension & $632(59.7)$ & $247(63.4)$ & $385(57.6)$ & 0.039 \\
\hline Prior myocardial infarction & $60(5.7)$ & $18(4.6)$ & $42(6.3)$ & 0.296 \\
\hline Congestive heart failure & $170(16.1)$ & $53(13.6)$ & $117(17.5)$ & 0.100 \\
\hline Reduced LVEF at baseline & $138(13.2)$ & $46(12.1)$ & $92(13.9)$ & 0.448 \\
\hline Thromboembolic events & $84(7.9)$ & $23(5.9)$ & $61(9.1)$ & 0.076 \\
\hline $\mathrm{CHADS}_{2}$ score & $1.12 \pm 1.05$ & $1.08 \pm 0.95$ & $1.14 \pm 1.1$ & 0.418 \\
\hline Permanent AF & $500(47.3)$ & $189(48.5)$ & $311(46.6)$ & 0.566 \\
\hline AF-related hospitalizations & $3.1 \pm 2.9$ & $3.3 \pm 3.1$ & $3.1 \pm 2.7$ & 0.178 \\
\hline Symptomatic $\mathrm{AF}^{\mathrm{a}}$ & $938(88.7)$ & $336(86.2)$ & $602(90.1)$ & 0.052 \\
\hline Ventricular rate $>100 \mathrm{bpm}^{\mathrm{b}}$ & $223(21.1)$ & $87(22.3)$ & $136(20.4)$ & 0.364 \\
\hline Aspirin & $486(45.9)$ & $165(42.3)$ & $321(48.1)$ & 0.074 \\
\hline Oral vitamin $\mathrm{K}$ antagonist therapy & 401 (37.9) & $179(45.9)$ & $222(33.2)$ & $<0.001$ \\
\hline
\end{tabular}

Notes: Data are shown as the mean \pm standard deviation or number and percent. ${ }^{\mathrm{a}} \mathrm{AF}-$ related symptoms at baseline; boccasional fast ventricular rate during $\mathrm{AF}$ at any point during follow-up.

Abbreviations: bpm, beats per minute; LVEF, left ventricular ejection fraction; CHADS, congestive heart failure, hypertension, age $\geq 75$ years, diabetes mellitus, prior stroke or transient ischemic attack; AF, atrial fibrillation. 
Table 2 Patients' self-reported social characteristics in the questionnaire

\begin{tabular}{ll}
\hline Level of education & \\
Elementary school & $44(11.3 \%)$ \\
High school & $169(43.3 \%)$ \\
College/university & $177(45.4 \%)$ \\
Employment status & \\
Employed & $17 \mid(43.8 \%)$ \\
Retired & $219(56.2 \%)$ \\
Marital status* & \\
Without partner & $73(18.7 \%)$ \\
With partner & $316(81.2 \%)$ \\
Children* & \\
No & $4 I(10.5 \%)$ \\
Yes & $348(89.5 \%)$ \\
\hline
\end{tabular}

Notes: Data are shown as number and percent. *Data for one patient are missing.

AF-related symptoms over time, 113 patients $(29.0 \%)$ answered "No". Of 277 patients (71.0\%) whose answer was "Yes", 213 (54.6\%) thought the reduction of symptoms was due to treatment, 19 (4.9\%) attributed the improvement to "other reasons", and only $45(11.5 \%)$ claimed that they "got used" to AF over time. Of the latter 45 patients, 28 (62.2\%) had paroxysmal AF, 12 (26.7\%) had persistent $\mathrm{AF}$, and five (11.1\%) had permanent $\mathrm{AF}$ at the time of the questionnaire. Overall, 12 of 189 patients with permanent AF (6.3\%) and 40 of 201 with intermittent AF (19.9\%) had got used to their AF. Patients were also asked whether AF negatively influenced their career (or working capacity) and/or private life (Table 3); 267 patients (68.4\%) answered "Yes, my career", and 273 patients $(70.0 \%)$ thought that their private life was impaired (of note, 277 patients [71.0\%] answered "Yes, both"). Only 81 patients

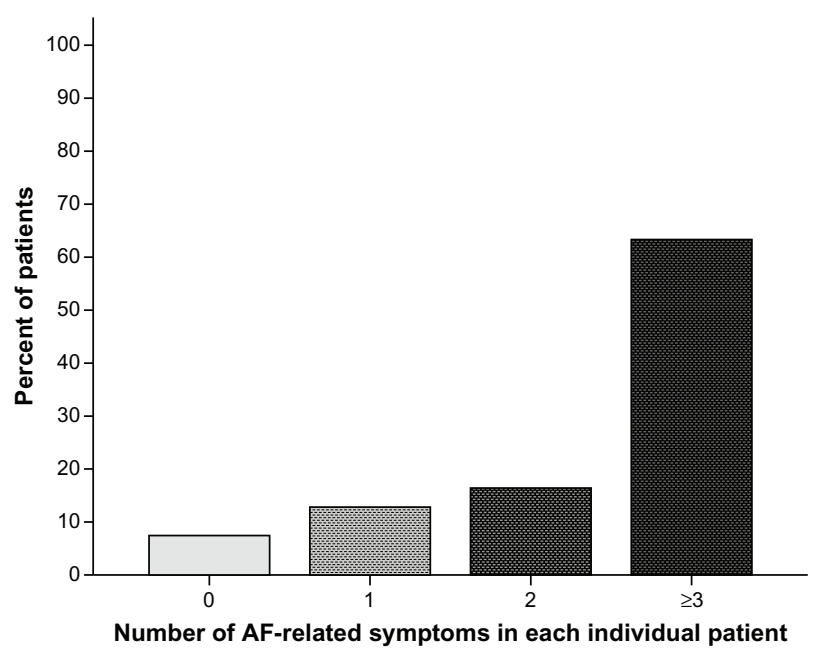

Figure I Patients' self-reported number of symptoms attributable to atrial fibrillation.

Abbreviation: $\mathrm{AF}$, atrial fibrillation.
(20.8\%) stated that AF did not influence either their career or private life.

The question "Do you agree the rhythm (ie, sinus rhythm or AF) is not important as long as the heart does not beat too fast?" was answered as follows: 201 patients $(51.5 \%)$ answered "No, I would always prefer the perception of being in sinus rhythm"; 137 patients (35.1\%) stated "Yes, I agree"; and 52 patients (13.4\%) chose "I do not know". When asked what they feared most regarding AF, 202 patients (51.8\%) chose "Irregular and/or occasionally fast heart beat", only 86 patients $(22.1 \%)$ were concerned about AF-related complications including ischemic stroke, whilst 102 patients (26.1\%) stated "I do not know" (Table 3). Only 208 patients $(53.3 \%)$ stated that they could identify their current heart rhythm (ie, sinus rhythm or AF) reliably, but 62/208 patients (29.8\%) did not correctly identify their true heart rhythm.

Finally, when patients were asked if they would accept any invasive procedure even if risky and/or painful if it could possibly cure their AF, 181 patients (46.4\%) answered "Yes", 99 (25.4\%) chose "Most probably yes", and 110 (28.2\%) stated that they would not accept an invasive procedure even if it was likely to cure their AF (Table 3).

On univariate analysis, male gender, age at baseline, permanent AF, self-reported AF symptom score, and

Table 3 AF-related questions and patients' answers

\begin{tabular}{|c|c|c|c|}
\hline Q: & \multicolumn{3}{|c|}{ Was there any reduction in AF-related symptoms over time? } \\
\hline A: & No & 113 & $29.0 \%$ \\
\hline & Yes & 277 & $71.0 \%$ \\
\hline & Yes, due to treatment & 213 & $54.6 \%$ \\
\hline & Yes, due to other reasons & 19 & $4.9 \%$ \\
\hline & Yes, "got used" to AF & 45 & $11.5 \%$ \\
\hline Q: & \multicolumn{3}{|c|}{$\begin{array}{l}\text { Do you think AF negatively influences your career (or working } \\
\text { capacity) and/or your private life? }\end{array}$} \\
\hline A: & Yes, my career & 267 & $68.4 \%$ \\
\hline & Yes, my & 273 & $70.0 \%$ \\
\hline & None & 81 & $20.8 \%$ \\
\hline Q: & \multicolumn{3}{|c|}{$\begin{array}{l}\text { Do you agree the rhythm (that is, SR or AF) is not important as } \\
\text { long as the heart does not beat too fast? }\end{array}$} \\
\hline A: & No, I prefer sinus rhythm & 201 & $51.5 \%$ \\
\hline & Yes, I agree & 137 & $35.1 \%$ \\
\hline & I do not know & 52 & $13.3 \%$ \\
\hline Q: & \multicolumn{3}{|l|}{ What do you fear most regarding AF? } \\
\hline A: & Irregular and/or occasionally fast heart beating & 202 & $51.8 \%$ \\
\hline & AF-related complications including ischemic stroke & 86 & $22.1 \%$ \\
\hline & I do not know & 102 & $26.1 \%$ \\
\hline Q: & \multicolumn{3}{|c|}{$\begin{array}{l}\text { Would you accept any invasive procedure, even if painful and/or } \\
\text { risky, if it could possibly cure your AF? }\end{array}$} \\
\hline A: & No & 110 & $28.2 \%$ \\
\hline & Yes & 181 & $46.4 \%$ \\
\hline & Yes, most probably & 99 & $25.4 \%$ \\
\hline
\end{tabular}

Note: Data are shown as the number and percent.

Abbreviations: AF, atrial fibrillation; $Q$, question; $A$, answer; $S R$, sinus rhythm. 
impaired career/working capacity were significantly associated with a positive answer (all $P<0.05$ ). In the multivariable analysis, male gender and impaired career/ working capacity were independent predictors of a positive attitude towards invasive treatment of AF (relative risk $2.1,95 \%$ confidence interval $[\mathrm{CI}] 1.3-3.4, P=0.002$ and relative risk $2.2,95 \% \mathrm{CI} 1.4-3.3, P<0.001$, respectively, Table 4).

On the other hand, female gender, increasing age at baseline, permanent AF, a high level of education (college/ university), retirement, living with a partner, and career/working capacity or private life not being impaired by AF were significantly related to a negative answer in the univariate analyses (all $P<0.05$ ). In multivariable analysis, female gender (relative risk 2.6, 95\% CI 1.5-4.4, $P<0.001$ ), increasing age at baseline (relative risk 1.3, 95\% CI 1.1-1.6, $P=0.011$ ), permanent AF (relative risk 2.8, 95\% CI 1.7-4.5, $P<0.001$ ), college/university level of education (relative risk $1.8,95 \%$ CI 1.1-3.0, $P=0.019)$, and private life not being affected by AF (relative risk 2.4, 95\% CI 1.4-3.9, $P=0.001$ ) were independent predictors of a negative attitude towards an invasive procedure for AF (Table 4).

Underlying cardiac comorbidities, left ventricular systolic function, $\mathrm{CHADS}_{2}$ score, clinical type of AF, AF symptoms, number of AF-related hospitalizations during follow-up, and use of oral anticoagulants or aspirin were not significantly associated with patient attitudes towards invasive treatment of AF (all $P>0.05$ ).

\section{Discussion}

This study shows that only a small percentage of patients really do accommodate to AF over time despite active long-term treatment, whilst the majority of AF patients still prefer what they perceive as sinus rhythm even at the cost of accepting an invasive treatment procedure. The patients' preferences are not related to the severity of symptoms or the actual presence of AF (indeed, many patients could not identify their current heart rhythm accurately). These findings could have relevant clinical implications.

The present study included predominantly middle-aged and relatively healthy AF patients who had already undergone an average 10-year period of active treatment starting from their initial diagnosis of AF, and were seen regularly by their cardiologists who encouraged them to ask questions and participate actively in the treatment of their condition. The most striking findings in our study were that only $12 \%$ of patients stated that they had got used to AF over time and as many as three quarters of them would undergo an invasive procedure for AF, either immediately or after being supplied with additional information. Indeed, it is well known that AF patients have a significantly impaired quality of life compared with healthy controls, the general population, and patients with coronary heart disease, ${ }^{3,16}$ but it would be intuitively expected that the majority of AF patients would accommodate to a chronic illness over time, particularly with an active and mostly satisfactory treatment. Although randomized trials have documented improvement in quality

Table 4 Univariate and multivariable predictors of patient attitudes towards an invasive treatment of AF

\begin{tabular}{|c|c|c|c|c|c|c|}
\hline \multirow[t]{2}{*}{ Variables } & \multicolumn{3}{|c|}{ Univariate analysis } & \multicolumn{3}{|c|}{ Multivariable analysis } \\
\hline & HR & $95 \% \mathrm{Cl}$ & P-value & HR & $95 \% \mathrm{Cl}$ & P-value \\
\hline \multicolumn{7}{|c|}{ "Yes" to intervention (would you accept any invasive procedure, even if risky and/or painful, if it could possibly cure your AF?) } \\
\hline Male gender & 1.91 & $1.22-2.99$ & 0.005 & 2.11 & $1.33-3.38$ & 0.002 \\
\hline Age at baseline (decades) & 0.82 & $0.69-0.97$ & 0.019 & 0.83 & $0.70-0.99$ & 0.041 \\
\hline Permanent AF & 0.64 & $0.43-0.96$ & 0.030 & - & - & - \\
\hline Self-reported AF symptom score & 2.28 & $1.79-2.91$ & $<0.001$ & - & - & - \\
\hline Negatively influenced career & 1.93 & $1.28-2.90$ & 0.002 & 2.16 & $1.4 \mathrm{I}-3.30$ & $<0.001$ \\
\hline Negatively influenced private life & 1.57 & $1.04-2.37$ & 0.031 & - & - & - \\
\hline \multicolumn{7}{|c|}{ "No" to intervention (would you accept any invasive procedure, even if painful and/or risky, if it could possibly cure your AF?) } \\
\hline Female gender & 2.16 & $1.35-3.44$ & 0.001 & 2.60 & $1.55-4.37$ & $<0.001$ \\
\hline Age at baseline (decades) & $\mathrm{I} .4 \mathrm{I}$ & $1.16-1.71$ & 0.001 & 1.32 & $1.07-1.63$ & 0.011 \\
\hline Permanent AF & 2.95 & $1.85-4.69$ & $<0.001$ & 2.75 & $1.68-4.49$ & $<0.001$ \\
\hline College/university education & 1.59 & $1.02-2.48$ & 0.041 & 1.81 & $1.10-2.97$ & 0.019 \\
\hline Retired & 1.91 & $1.21-3.04$ & 0.006 & - & - & - \\
\hline Married/with partner & 0.52 & $0.31-0.89$ & 0.017 & - & - & - \\
\hline Career not influenced by AF & 2.00 & $1.26-3.17$ & 0.003 & - & - & - \\
\hline Private life not influenced by AF & 2.37 & $1.49-3.77$ & $<0.001$ & 2.37 & $1.43-3.92$ & 0.001 \\
\hline
\end{tabular}

Notes: All variables stated in Tables I and 2 were tested in the univariate analysis. Only variables with a statistically significant relationship with the patients' answers are shown in Table 4.

Abbreviations: $\mathrm{AF}$, atrial fibrillation; $\mathrm{Cl}$, confidence interval; $\mathrm{HR}$, hazards ratio. 
of life with both rhythm control and rate control, patients in the relevant trials were not asked directly if they had accommodated to AF. ${ }^{3,7,20-22}$ It has been shown that up to one third of AF patients may suffer from persistent depression, ${ }^{23}$ and more than one third of patients from the AF-AWARE survey were still worried or afraid about their AF for on average 8 years after the first appearance of symptoms and 2.6 years following the clinical diagnosis of AF. ${ }^{16}$

Patient preferences in our study were not in line with their judgment about the effect of AF treatment on symptom relief, with $71 \%$ of patients stating that they have experienced a reduction in their AF-related symptoms over time, with more than one half of these patients attributing this to the effect of AF treatment. An essentially similar paradox was recorded in the AF-AWARE survey, in which there was a high level of patient satisfaction with AF treatment, but many patients were still substantially concerned about having AF. ${ }^{16}$ Indeed, when patients in our study were asked to agree with the statement that heart rhythm is not important as long as the heart does not beat too fast, only one third of them answered "Yes", whilst more than one half of patients clearly stated they preferred the perception of being in sinus rhythm. At the same time, $63 \%$ of patients in our study were not able to state their current heart rhythm or identify it incorrectly. Thus, patient preferences could well result from their expectations of treatment (which could also be influenced by the physician's efforts to achieve rhythm control) rather than being a direct reaction to the rhythm disorder itself. Overall, it is likely that, in many AF patients, a substantial dissociation exists between the "resignation to fate" in the absence of more efficient methods for the treatment of AF and their true preferences if they could choose without restrictions.

The concept that AF could be a readily modifiable risk factor for cardiovascular morbidity and mortality might be facilitated by continuous development and increasing availability of AF catheter ablation techniques for various types of AF patients, including those with AF of longer duration or with more comorbidities, although it is still not proven that treatment of the rhythm disorder prolongs survival in patients with AF. ${ }^{13,24}$ Several observational studies have suggested lower mortality and adverse cardiovascular event rates in AF patients treated with ablation, ${ }^{25,26}$ along with a number of randomized trials investigating whether such invasive rhythm control can reduce cardiovascular morbidity and mortality and improve the maintenance of sinus rhythm, including the Radiofrequency Ablation versus Antiarrhythmic drugs for atrial Fibrillation Treatment study (RAAFT), the Catheter ABlation versus ANti-arrhythmic drug therapy for Atrial fibrillation trial (CABANA), and the Early treatment of Atrial fibrillation for Stroke prevention Trial (EAST). These three trials are registered at clinicaltrials.gov (NCT00392054, ${ }^{27}$ NCT00911508, ${ }^{28}$ and NCT01288352, ${ }^{29}$ respectively).

Up to three quarters of patients in our study would choose an invasive AF treatment, regardless of AF symptom severity and underlying comorbidities. Not surprisingly, younger age and impaired career or working capacity were independent predictors of such an attitude, whilst older age, permanent $\mathrm{AF}$, and an unaffected private life predicted a preference for noninvasive treatment. However, we found a significant gender-related difference in patient preference, in that male gender was an independent predictor of choosing an invasive treatment option whilst female gender predicted choosing a noninvasive therapy. Indeed, a recent study reported that women had a lower overall success rate with AF ablation compared with men because of fewer repeat ablations due to patient choice (the success rates of initial and repeat ablation, when performed, were similar in both genders). ${ }^{30}$ On the other hand, it has been well documented that women with AF are more symptomatic than their male counterparts. ${ }^{19,31}$

In our study, a high level of patient education was an independent predictor of favoring a noninvasive AF treatment. A recent report demonstrated that higher education predicted a better understanding of AF by patients. ${ }^{32}$ The patients in our study with a high level of education could probably better appreciate the AF treatment goals and were more realistic about treatment efficacy long after following the first occurrence of $\mathrm{AF}$.

In line with other reports, the patients' perception that AF confers an increased risk of complications, including ischemic stroke, was not at a satisfactory level in our study, ${ }^{16}$ with less than a quarter of patients stating that they feared AF-related complications, including ischemic stroke, whilst more than a half of them were afraid of irregular or fast heart beats. Although our patients were regularly counseled by a cardiologist, the risk of stroke and other AF complications perhaps seemed too abstract in relation to the patients' current perception of priorities.

\section{Study limitations}

The present study is limited by its single-center, observational design, although it was conducted in a tertiary health center which serves all parts of the country. In addition, we did not use a standardized, previously validated questionnaire, and the physicians' active efforts towards successful long-term rhythm control could influence the patients' perception of a more favorable strategy for AF manage- 
ment. On the other hand, patients were regularly seen by a cardiologist over a long period and were repeatedly offered explanations and reassurance regarding the nature of the disease and the advantages (and disadvantages) of the AF treatment options available, in line with contemporary international recommendations for AF management. In addition, the choice of words in some questions (or answers offered) could affect the patients' responses. For example, the question of whether a patient would accept any invasive procedure, even if painful and/or risky, if it could possibly cure AF, could be highly prejudicial with respect to patient expectation. However, a detailed explanation of AF catheter ablation (including published success rates with the procedure) was given to patients in our study in 2005, two years prior to the questionnaire (at that time, preparations for $\mathrm{AF}$ radiofrequency catheter ablation had started in our hospital) and during follow-up, although AF catheter ablation was still not available at the time of questionnaire. Due to the relatively small number of patients included in our survey, the results should be interpreted with some caution. In addition, despite random patient selection, there might be a selection bias, given that approximately one third of patients from the cohort with newly diagnosed AF were included in the survey. However, there were no profound differences in clinical characteristics between the questionnaire group and the rest of the cohort. Due to the registry-based design of our study, detailed data on the frequency (and quality) of International Normalized Ratio control were not available, and the difficulties associated with long-term treatment using vitamin $\mathrm{K}$ antagonists might have influenced patient preferences in our study. However, oral anticoagulant therapy was not significantly associated with a response of "Yes" to an invasive AF treatment (hazards ratio 0.92, 95\% CI $0.62-1.37, P=0.673$ ).

In conclusion, our findings suggest that most AF patients would prefer sinus rhythm, regardless of time from the initial diagnosis of AF, severity of AF-related symptoms, and underlying comorbidities, and would readily accept an invasive procedure for AF treatment if it could probably cure their AF, even at the price of possible periprocedural complications and/or procedure-related discomfort. To satisfy the highly motivated attitude of patients towards restoration and maintenance of sinus rhythm, ongoing efforts are needed to establish optimal strategies for treatment of AF.

\section{Disclosure}

The authors report no conflicts of interest in this work.

\section{References}

1. Benjamin EJ, Wolf PA, D'Agostino RB, Silbershatz H, Kannel WB, Levy D. Impact of atrial fibrillation on the risk of death: the Framingham Heart Study. Circulation. 1998;98:946-952.

2. Luderitz B, Jung W. Quality of life in patients with atrial fibrillation. Arch Intern Med. 2000;160:1749-1757.

3. Thrall G, Lane D, Carrol D, Lip GYH. Quality of life in patients with atrial fibrillation: a systematic review. Am J Med. 2006;119:448. e1-e448. e19.

4. Wyse DG, Waldo AL, DiMarco JP, et al; Atrial Fibrillation Follow-up Investigation of Rhythm Management (AFFIRM) Investigators. A comparison of rate control and rhythm control in patients with atrial fibrillation. N Engl J Med. 2002;347:1825-1833.

5. Van Gelder IC, Hagens VE, Bosker HA, et al; Rate Control versus Electrical Cardioversion for Persistent Atrial Fibrillation Study Group. A comparison of rate control and rhythm control in patients with recurrent persistent atrial fibrillation. N Engl J Med. 2002;347:1834-1840.

6. Hohnloser SH, Kuck KH, Lilienthal J. Rhythm or rate control in atrial fibrillation - Pharmacological Intervention in Atrial Fibrillation (PIAF): a randomised trial. Lancet. 2000;356:1789-1794.

7. Carlsson J, Miketic S, Windeler J, et al; STAF Investigators. Randomized trial of rate-control versus rhythm-control in persistent atrial fibrillation: the Strategies of Treatment of Atrial Fibrillation (STAF) study. J Am Coll Cardiol. 2003;41:1690-1696.

8. Testa L, Biondi-Zoccai GG, Dello Russo A, Belloci F, Andreotti F, Crea F. Rate-control vs rhythm-control in patients with atrial fibrillation: a meta-analysis. Eur Heart J. 2005;26:2000-2006.

9. Roy D, Talajic M, Nattel S, et al; Atrial Fibrillation and Congestive Heart Failure Investigators. Rhythm control versus rate control for atrial fibrillation and heart failure. N Engl J Med. 2008;358:2667-2677.

10. Corley SD, Epstein AE, DiMarco JP, et al; AFFIRM Investigators. Relationships between sinus rhythm, treatment, and survival in the Atrial Fibrillation Follow-Up Investigation of Rhythm Management (AFFIRM) Study. Circulation. 2004;109:1509-1513.

11. Ionescu-Ittu R, Abrahamowicz M, Jackevicius CA, et al. Comparative effectiveness of rhythm control vs rate control drug treatment effect on mortality in patients with atrial fibrillation. Arch Intern Med. 2012;172: 997-1004.

12. Kumagai K. Catheter ablation of atrial fibrillation. State of the Art. Circ J. 2011;75:2305-2311.

13. Nault I, Miyazaki S, Forclaz A, et al. Drugs vs ablation for the treatment of atrial fibrillation: the evidence supporting catheter ablation. Eur Heart J. 2010;31:1046-1057.

14. Camm AJ, Lip GYH, De Caterina R, et al. 2012 Focused Update of the ESC Guidelines for the Management of Atrial Fibrillation. Eur Heart J. 2012;33:2719-2747.

15. Camm JA, Breithardt G, Crijns H, et al. Real-life observations of clinical outcomes with rhythm- and rate-control therapies for atrial fibrillation. RECORDAF (Registry on Cardiac Rhythm Disorders Assessing the Control of Atrial Fibrillation). J Am Coll Cardiol. 2011;58: 493-501.

16. Aliot E, Brethardt G, Brugada J, et al; for the Atrial Fibrillation Awareness And Risk Education (AF AWARE) group [comprising the Atrial Fibrillation Association (AFA), the European Heart Rhythm Association (EHRA), Stroke Alliance for Europe (SAFE), and the World Heart Federation (WHF)]. An international survey of physician and patient understanding, perception, and attitudes to atrial fibrillation and its contribution to cardiovascular morbidity and mortality. Europace. 2010;12:626-633.

17. Potpara TS, Vasiljevic ZM, Vujisic-Tesic BD, et al. Mitral annular calcification predicts cardiovascular morbidity and mortality in middle-aged patients with atrial fibrillation: the Belgrade Atrial Fibrillation Study. Chest. 2011;140:902-910.

18. Potpara TS, Stankovic GR, Beleslin BD, et al. A 12-year follow-up study of patients with newly-diagnosed lone atrial fibrillation: implications of arrhythmia progression on prognosis: the Belgrade Atrial Fibrillation Study. Chest. 2012;141:339-347. 
19. Potpara TS, Marinkovic JM, Polovina MM, et al. Gender-related differences in presentation, treatment and long-term outcome in patients with first-diagnosed atrial fibrillation and structurally normal heart: the Belgrade Atrial Fibrillation Study. Int J Cardiol. 2012;161:39-44.

20. Gronefeld GC, Lilienthal J, Kuck KH, Hohnloser SH. Pharmacological Intervention in Atrial Fibrillation (PIAF) Study investigators. Impact of rate versus rhythm control on quality of life in patients with persistent atrial fibrillation. Eur Heart J. 2003;24:1430-1436.

21. Hagens VE, Ranchor AV, Van Sonderen E, et al; RACE Study Group. Effect of rate or rhythm control on quality of life in persistent atrial fibrillation. Results from the Rate Control Versus Electrical Cardioversion (RACE) study. J Am Coll Cardiol. 2004;43:241-247.

22. Jenkins LS, Brodsky M, Schron E, et al. Quality of life in atrial fibrillation: the Atrial Fibrillation Follow-up Investigation of Rhythm Management (AFFIRM) study. Am Heart J. 2005;149:112-120.

23. Thrall G, Lip GY, Carroll D, Lane D. Depression, anxiety, and quality of life in patients with atrial fibrillation. Chest. 2007;132:1259-1264.

24. Van Gelder IC, Haegeli LM, Brandes A, et al. Rationale and current perspective for early rhythm control therapy in atrial fibrillation. Europace. 2011;13:1517-1525.

25. Bunch TJ, Crandall BG, Weiss JP, et al. Patients treated with catheter ablation for atrial fibrillation have long-term rates of death, stroke, and dementia similar to patients without atrial fibrillation. J Cardiovasc Electrophysiol. 2011;22:839-845.

26. Lin YJ, Chao TF, Tsao HM, et al. Successful catheter ablation reduces the risk of cardiovascular events in atrial fibrillation patients with CHA2DS2-VASc risk score of 1 and higher. Europace. 2013;15: 676-684.
27. Population Health Research Institute . First Line Radiofrequency Ablation Versus Antiarrhythmic Drugs for Atrial Fibrillation Treatment (The RAAFT Study). Available from: http:/clinicaltrials.gov/show/ NCT00392054. NLM identifier: NCT00392054. Accessed August 16, 2013.

28. Mayo Clinic. Catheter Ablation vs Anti-arrhythmic Drug Therapy for Atrial Fibrillation Trial (CABANA). Available from: http://clinicaltrials. gov/ct2/show/NCT00911508. NLM identifier: NCT00911508. Accessed August 16, 2013.

29. German Atrial Fibrillation Network. Early Treatment of Atrial Fibrillation for Stroke Prevention Trial (EAST). Available from: http:// clinicaltrials.gov/show/NCT01288352. NLM identifier: NCT01288352. Accessed August 16, 2013.

30. Winkle RA, Mead RH, Engel G, Patrawala RA. Long-term results of atrial fibrillation ablation: the importance of all initial ablation failures undergoing a repeat ablation. Am Heart J. 2011;162:193-200.

31. Dagres N, Nieuwlaat R, Vardas PE, et al. Gender-related differences in presentation, treatment, and outcome of patients with atrial fibrillation in Europe. A report from the Euro Heart Survey on Atrial Fibrillation. J Am Coll Cardiol. 2007;49:572-577.

32. $\mathrm{Xu} \mathrm{W}$, Sun G, Lin Z, et al. Knowledge, attitude, and behavior in patients with atrial fibrillation undergoing radiofrequency catheter ablation. J Interv Card Electrophysiol. 2010;28:199-207.
Patient Preference and Adherence

\section{Publish your work in this journal}

Patient Preference and Adherence is an international, peer-reviewed, open access journal focusing on the growing importance of patient preference and adherence throughout the therapeutic continuum. Patient satisfaction, acceptability, quality of life, compliance, persistence and their role in developing new therapeutic modalities and compounds to

\section{Dovepress}

optimize clinical outcomes for existing disease states are major areas of interest. This journal has been accepted for indexing on PubMed Central. The manuscript management system is completely online and includes a very quick and fair peer-review system. Visit http://www.dovepress.com/ testimonials.php to read real quotes from published authors. 